\title{
Heartbeat: sudden cardiac death risk predicted by simple ECG measures
}

Sudden cardiac death (SCD) accounts for $10 \%-20 \%$ of all deaths in high-income countries, with about $50 \%$ of victims having no prior history of heart disease. Currently, primary prevention of SCD is limited to patients with known left ventricular dysfunction or an underlying cardiac arrhythmia. However, there is no simple method for identifying apparently healthy people who have a high risk of SCD. Based on ECG and outcome data in 6830 subjects in the Mini-Finland Health Study, Holkeri and colleagues ${ }^{1}$ identified five simple abnormalities on a baseline ECG that were independently associated with SCD risk: heart rate $>80$ beats $/ \mathrm{min}, \mathrm{PR}$ duration $>220 \mathrm{~ms}$, QRS duration $>110 \mathrm{~ms}$, left ventricular hypertrophy and T-wave inversion. In a separate validation cohort of 10617 subjects, the presence of 1,2 or 3 of these abnormalities was associated with a progressive increase in SCD risk with an HR of 10.82 (95\% CI 3.23 to 36.25 ) for patients with 3 of more of these ECG findings compared with those with no ECG abnormalities (figure 1).

In the accompanying editorial, Sinner et $a l^{2}$ comment that 'the presented study constitutes an important step towards improved SCD risk prediction with the advantage that 'the selected ECG markers can be determined in almost any clinical setting, thereby facilitating potential broad application of the score.' However, prospective randomized clinical studies are needed because 'Individuals at increased risk for SCD might need to be subjected to intensified monitoring, intensified medical treatment, or-ultimately-implantation of an ICD."

Appropriateness criteria and other guidelines seek to improve patient care by identifying which patients will benefit from a procedure, such as percutaneous coronary intervention (PCI), while reducing healthcare system costs by reducing unnecessary procedures. However, benefit often is defined from the physician point of view with few studies reporting patient reported outcome measures (PROMs) in patients undergoing elective PCI. In

Correspondence to Professor Catherine M Otto, Division of Cardiology, University of Washington, Seattle,WA 98195, USA; cmotto@uw.edu
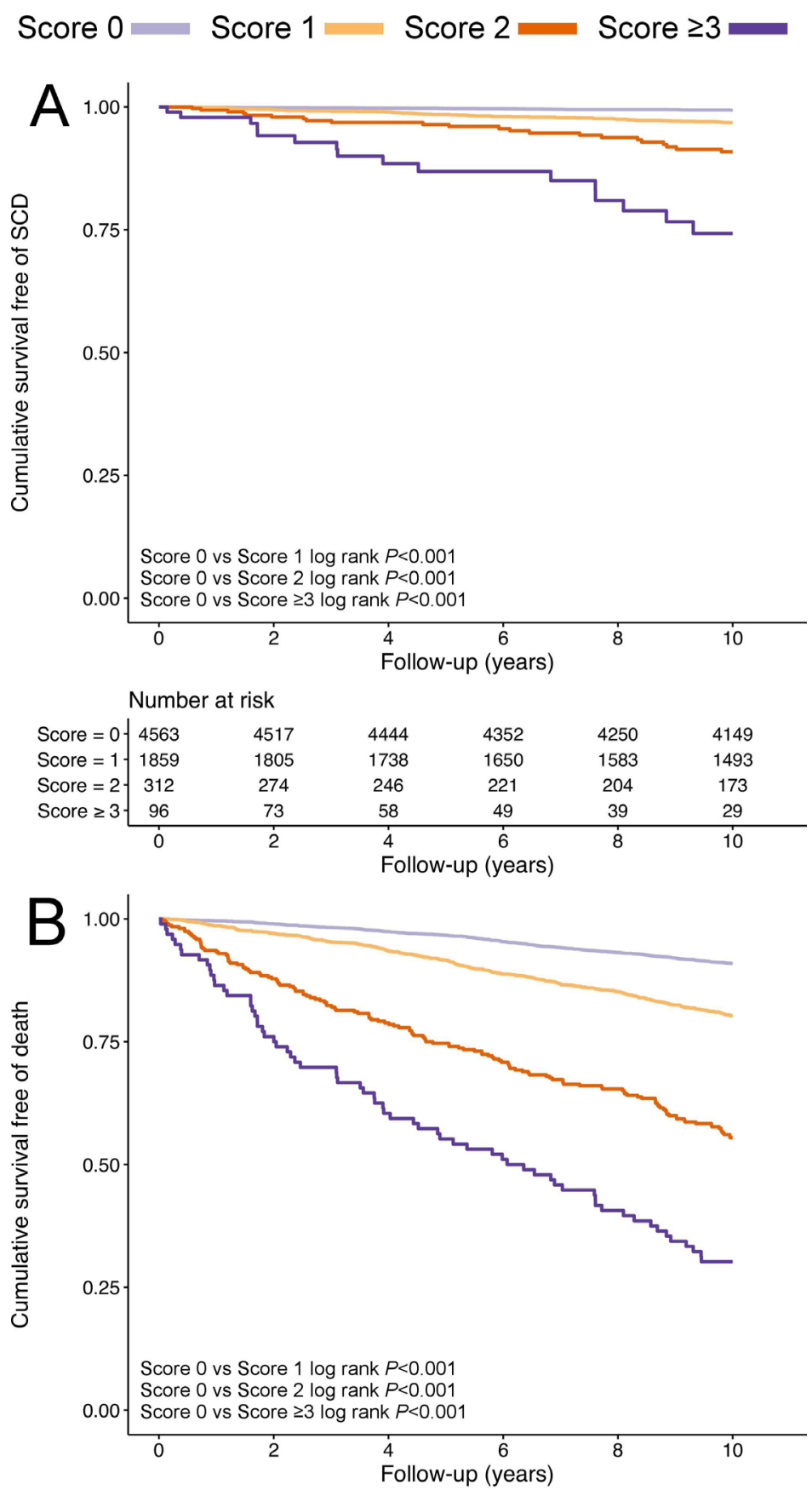

Number at risk

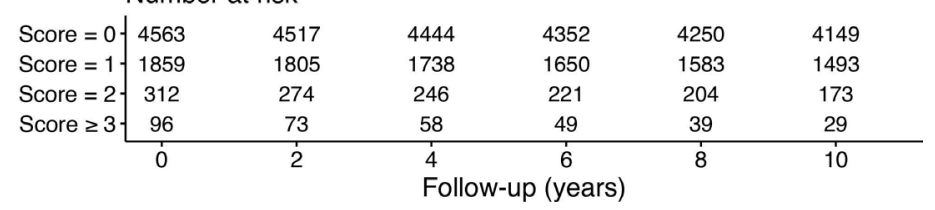

Figure 1 Kaplan-Meier survival plots for (A) SCD and (B) death from any cause according to the ECG risk score in the 10-year follow-up. SCD, sudden cardiac death. 


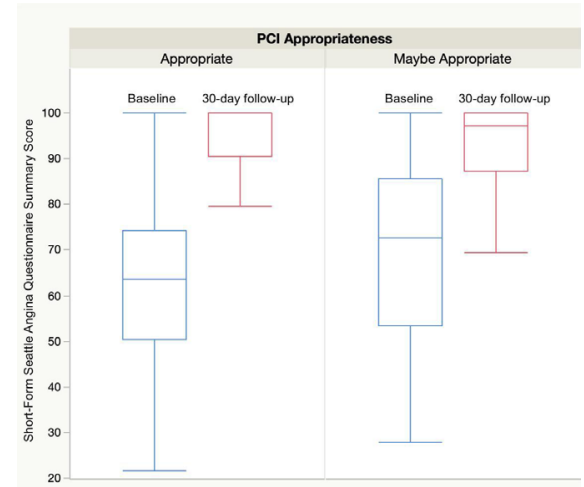

Figure 2 Boxplots demonstrating change in the Short-Form Seattle Angina Questionnaire summary score by $\mathrm{PCl}$ appropriateness. Boxplots indicate the median and IQR for values of the Short-Form Seattle Angina Questionnaire summary score at baseline and 30-day follow-up according to $\mathrm{PCI}$ appropriateness. The Short-Form Seattle Angina Questionnaire-7 is scaled from 0 to 100 , with lower scores indicating more severe angina. $\mathrm{PCl}$, percutaneous coronary intervention.

a study in this issue of Heart, Yang and colleagues $^{3}$ found that patients undergoing PCI has a similar improvement in angina, as measured by PROMs, regardless of whether PCI was considered appropriate by current criteria (figure 2).

In an editorial discussing these findings, Arnold ${ }^{4}$ points out that 'patients' symptom burden may be a better measure of appropriateness of PCI' than a reduction in ischaemia risk, as measured by stress testing or imaging. Thus, ensuring clinical benefits depends on using PROMs that reliably assess patients' symptom burden and integrating these PROMs into routine clinical practice. In a second editorial, Bradley ${ }^{5}$ suggests a 'cloudy future for appropriateness criteria' as currently defined. Instead 'we need to put patient-reported health status front and centre in clinical settings where the anticipated benefit is not the reduction of mortality or other clinical events. This includes stratifying patients on the basis of health status deficit at the time of randomisation in clinical trials, and routine capture of health status in clinical registries and clinical care.'

Coronary artery vasculitis due to Kawasaki disease typically starts in childhood but clinical sequelae occur over the patient's lifetime. Up to $2 / 3$ of patients suffer major adverse cardiovascular events within 30 years of diagnosis due to the lifelong risk of coronary thrombosis, coronary stenosis and acute coronary syndromes. Practical guidance for management of these patients is provided in a consensus statement published in this issue of Heart, with contributions from multiple professional societies. $^{6}$ Tables summarise the classification of risk for coronary dilation or aneurysm and provide recommendations for periodic follow-up assessments based on clinical, ECG, imaging and blood tests as well as psychological support and lifestyle issues. Detailed flowcharts highlight the atypical presentation of acute coronary syndromes and the unique aspects of emergency management in patients with previous Kawasaki disease.

An outstanding review article in this issue of Heart provides an update on

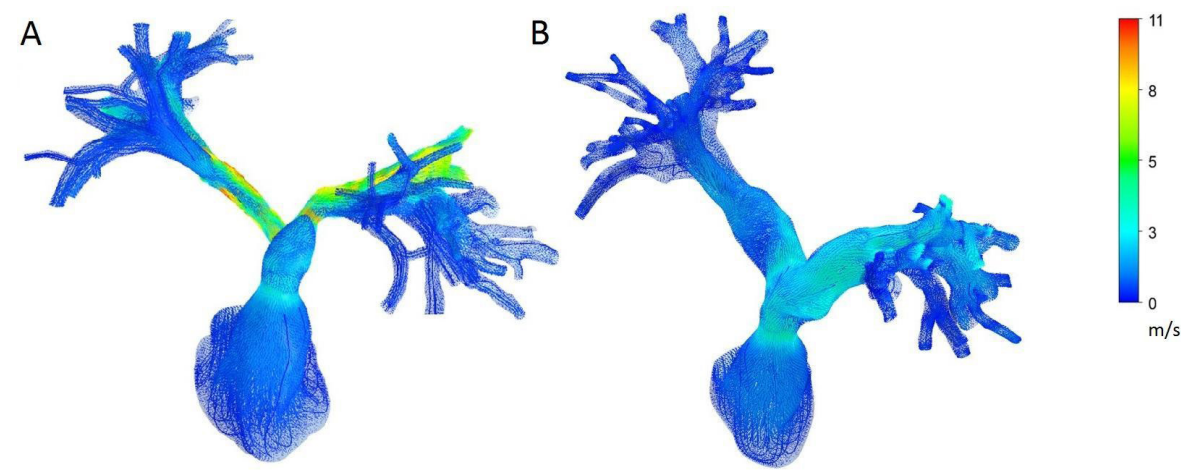

Figure 3 Visualisation of flow pathlines and vector fields of computational fluid dynamics analyses in a toddler with valvular and supravalvular pulmonary stenosis. (A) The situation before Y-stent implantation: multilevel stenosis of the pulmonary arteries resulting in flow acceleration in both branches. (B) The situation after Y-stent procedure. An increase in vessel diameter results in reduction of flow velocities. Image courtesy of Maartje Conijn, University Medical Center Utrecht. the use of three-dimensional and fourdimensional (3D plus time) flow assessment in patients with congenital heart disease (CHD). ${ }^{7}$ The abnormal flow patterns associated with native and surgically corrected CHD provide insights into the pathophysiology of disease and may allow development of better corrective procedures in the future using flow visualisation to restore normal flow patterns and minimise long-term adverse outcomes (figure 3).

In our Cardiology in Focus article, Qasim ${ }^{8}$ shares his experiences in founding a medical knowledge platform for sharing clinical cases and provides his top tips for cardiologists aspiring to become a health tech entrepreneur.

\section{Contributors All authors contributed.}

Funding The authors have not declared a specific grant for this research from any funding agency in the public, commercial or not-for-profit sectors.

Competing interests None declared.

Patient consent for publication Not required.

Provenance and peer review Commissioned; internally peer reviewed.

(C) Author(s) (or their employer(s)) 2020. No commercial re-use. See rights and permissions. Published by BMJ.

\section{Check for updates}

To cite Otto CM. Heart 2020;106:401-402.

Heart 2020;106:401-402.

doi:10.1136/heartjnl-2020-316698

ORCID iD

Catherine M Otto http://orcid.org/0000-0002-05279392

\section{REFERENCES}

1 Holkeri A, Eranti A, Haukilahti MAE, et al. Predicting sudden cardiac death in a general population using an electrocardiographic risk score. Heart 2020;106:427-33.

2 Sinner MF, Rizas KD, Kääb S. Keep it simple: the ECG and sudden cardiac death risk. Heart 2020;106:403-4.

3 Yang JX, Stevenson MJ, Valsdottir L, et al. Association between procedure appropriateness and patientreported outcomes after percutaneous coronary intervention. Heart 2020;106:441-6.

4 Arnold SV. Can we improve the appropriateness of $\mathrm{PCI}$ ? Heart 2020;106:405-6.

5 Bradley SM. Cloudy forecast for appropriate use criteria. Heart 2020;106:407-8.

6 Brogan P, Burns JC, Cornish J, et al. Lifetime cardiovascular management of patients with previous Kawasaki disease. Heart 2020;106:411-20.

7 Warmerdam E, Krings GJ, Leiner T, et al. Threedimensional and four-dimensional flow assessment in congenital heart disease. Heart 2020;106:421-6.

8 Qasim A. From finding sports partners to medical case sharing: transforming ideas into reality. Heart 2020;106:475-6. 